\title{
Generating optical orbital angular momentum at visible wavelengths using a plasmonic metasurface
}

\begin{abstract}
Ebrahim Karimi $^{1}$, Sebastian A Schulz ${ }^{1}$, Israel De Leon ${ }^{1}$, Hammam Qassim ${ }^{1}$, Jeremy Upham ${ }^{1}$ and Robert W Boyd $^{1,2}$
Light beams with a helical phase-front possess orbital angular momentum along their direction of propagation in addition to the spin angular momentum that describes their polarisation. Until recently, it was thought that these two 'rotational' motions of light were largely independent and could not be coupled during light-matter interactions. However, it is now known that interactions with carefully designed complex media can result in spin-to-orbit coupling, where a change of the spin angular momentum will modify the orbital angular momentum and vice versa. In this work, we propose and demonstrate that the birefringence of plasmonic nanostructures can be wielded to transform circularly polarised light into light carrying orbital angular momentum. A device operating at visible wavelengths is designed from a space-variant array of subwavelength plasmonic nano-antennas. Experiment confirms that circularly polarised light transmitted through the device is imbued with orbital angular momentum of $\pm 2 \hbar$ (with conversion efficiency of at least $1 \%$ ). This technology paves the way towards ultrathin orbital angular momentum generators that could be integrated into applications for spectroscopy, nanoscale sensing and classical or quantum communications using integrated photonic devices.
\end{abstract}

Light: Science \& Applications (2014) 3, e167; doi:10.1038/lsa.2014.48; published online 9 May 2014

Keywords: light orbital angular momentum; light spin angular momentum; plasmonic metasurface

\section{INTRODUCTION}

Spin angular momentum (SAM) and orbital angular momentum $(\mathrm{OAM})$ are associated with the polarisation and phase of the optical field, respectively. ${ }^{1}$ A striking difference between these momenta is the range of allowed values. SAM can be $\pm \hbar$ per photon, expressed as left or right circular polarisation, while OAM has an unbounded value of $\ell \hbar$ per photon, ${ }^{2} \ell$ being an integer. In an anisotropic and inhomogeneous medium, these otherwise independent momenta can be made to interact, changing both the polarisation and phase of the beam. ${ }^{3}$ This change depends on the incident beam's polarisation and the medium's topology stemming from its inhomogeneity. This relationship can be described by the Pancharatnam-Berry (geometrical) phase, ${ }^{4}$ and is what allows a beam to experience different optical paths associated with the trajectory of the polarisation evolution on the Poincare sphere. ${ }^{5}$ Recently, this phenomenon has enabled PancharatnamBerry phase optical elements: ${ }^{6,7}$ devices that control the output beam's wavefront according to the polarisation of the input beam. These devices could easily be inserted into the beam path of existing spectroscopic, nano-imaging or communication systems, as they do not rely on diffraction, adding OAM-based functionality that has the potential to distinguish between molecules of different chirality, enhance optical circular dichroism ${ }^{8}$ and encode multiple bits of information onto a single photon. ${ }^{9}$

Existing Pancharatnam-Berry phase optical elements include $q$ plates, ${ }^{3}$ made of liquid crystals, and computer-generated subwavelength gratings, ${ }^{6,10}$ made of micron-size dielectric features. These devices transform polarisation superposition states into complex structures rich in polarisation and phase singularities. ${ }^{11}$ However, the currently available technologies each have their respective limitations. Liquid crystal systems are highly susceptible to chemical degradation and feature a relatively large central singularity that limits their resolution. In the case of the gratings, their micron-size features prevent them from working in the visible regime. Moreover, their thicknesses prevent either system from operating with femtosecond pulses. Thus, there is a need for nanoscale, robust Pancharatnam-Berry phase optical elements capable of working in the visible spectrum.

In this work, we demonstrate a novel metasurface capable of optical spin-to-orbit coupling in the visible regime using a space-variant array of plasmonic gold nano-antennas with subwavelength thickness and a central singularity. Nano-fabrication advances have enabled the development of metamaterials capable of unconventionally controlling the flow of electromagnetic energy in the subwavelength domain. ${ }^{12,13}$ Recent demonstrations of ultrathin lenses, axicons ${ }^{14}$ and spiral phase plates ${ }^{15}$ use arrays of nano-antennas with different shapes to control the phase of optical fields. ${ }^{16}$ Particular arrangements of nanoparticles have also exhibited similar phase control. ${ }^{17,18}$ In such demonstrations, the optical field is manipulated locally by engineering the plasmonic resonances of individual nano-antennas such that the field experiences a different phase retardance at each nano-antenna. Thus, the desired optical device is designed by arranging different individual nano-antennas in the transverse plane. ${ }^{15}$ Instead, we propose an array of identical nano-antennas, possessing well-defined

${ }^{1}$ Department of Physics, University of Ottawa, Ottawa, Ont. K1N 6N5, Canada and ${ }^{2}$ Institute of Optics, University of Rochester, Rochester, NY 14627, USA

Correspondence: Dr E Karimi, Department of Physics, University of Ottawa, 150 Louis Pasteur, Ottawa, Ont. K1N 6N5, Canada

E-mail: ekarimi@uottawa.ca

Received 21 November 2013; revised 16 January 2014; accepted 19 January 2014 
topology, thus allowing us to control the beam's phase-front in the visible regime according to the Pancharatnam-Berry phase mechanism.

\section{MATERIALS AND METHODS}

First, we consider a metasurface consisting of a uniform, square lattice array of carefully designed, identical gold nano-antennas on a glass substrate. The nano-antenna dimensions and periodicity are chosen such that each element of the array exhibits a half-wavelength (i.e., $\pi$ ) optical retardation between two orthogonal linear polarisations. This metasurface is birefringent, since its action depends on the incident polarisation. For instance, it changes the helicity of normally incident, circularly polarised light, transforming left-circular polarisation into right-circular and vice versa. The light and array exchange SAM since the helicity of the input and the output photons are not identical, i.e., $\left|\Delta S^{z}\right|=\left|S_{\text {output }}^{z}-S_{\text {input }}^{z}\right|=2 \hbar \neq 0$. By conservation of angular momentum, the array should consequently spin about the beam's propagation axis. ${ }^{19}$ Although this rotation does not change the polarisation state of the output, the evolution trajectory of states on the polarisation Poincaré sphere does change. Thus, such a physical rotation introduces a global phase proportional to half of the solid angle enclosed by the polarisation trajectories (pole-to-pole) on the polarisation Poincaré sphere. ${ }^{5}$ This global phase is equal to $|2 \theta|$, where $\theta$ describes the angle over which the array has rotated. Next, we consider the particular case of a cylindrically symmetric metasurface possessing a transverse topological charge $q=1-$ meaning the nano-antenna orientation performs one full rotation along a path surrounding the origin. Because the metasurface is rotationally invariant about the plate origin, it does not exchange angular momentum with the optical field. Nonetheless, the helicities of incoming and outgoing beams are non-identical. Thus, the conservation of angular momentum dictates that the change in the SAM must be transformed into OAM.

For left and right circularly polarised input beams $\Delta S^{z}$ is $+2 \hbar$ and $-2 \hbar$ per photon, respectively. Therefore, the metasurface switches the polarisation of the incoming beam and induces an OAM of $\ell= \pm 2$, as schematically summarized in Figure 1 . This process is inherently geometry dependent and happens in a subwavelength scale. The intensity distribution of the emerging beam is no longer Gaussian: imprinting the helical phase-front changes it to a hypergeometric Gaussian distribution, which is not shape-invariant upon propagation. ${ }^{20}$ In an ideal, lossless structure, all light interacting with the nano-antennas would experience spin-to-orbit coupling. In a practical device, the conversion efficiency also depends on the uniformity of the nanoantennas' dimensions (i.e., consistency of phase retardation at a given wavelength) and the absorption coefficient of the metal.

We selected L-shaped nano-antennas for our realization because their dual resonances can be engineered to have equal amplitudes and a $\pi$ phase retardance between specific linear polarisations. The plasmonic nano-antenna array was modeled as gold on a glass substrate coated in indium tin oxide (ITO) using a fully vectorial Maxwell equation solver based on the finite difference time domain method (Lumerical FDTD 8). We assumed a perfectly periodic square array of nano-antennas by applying Bloch boundary conditions to the limits of the array's unit cell. The gold's material dispersion was taken into account by using frequency-dependent material permittivities. The permittivities of glass and gold were obtained from the Ref. 21, while that of ITO was obtained from spectroscopic ellipsometry measurements of the substrate sample. The excitation source was taken as a circularly polarised plane wave impinging at normal incidence onto the structure. For the calculations of the vector field amplitudes and phase retardance, the transmitted $E_{x}$ and $E_{y}$ field components are calculated after a propagation distance of $4 \mu \mathrm{m}$ in order to ensure a homogenous amplitude and phase distribution in the transverse direction. The dimensions of the antenna are designed such that the linear polarisation resonances of a nano-antenna (shown at top of Figure 2a) have a $\pi$-phase shift between them. Consequently, circular polarised beams (Figure $2 \mathrm{a}$ ), which can be described as equal amplitudes of the two linear polarisations, will be switched from one circular-handedness to the other by this $\pi$-phase shift. While this retardance of $\pi$ is possible between specific linear polarisations, this design exhibits slightly different retardance for circularly polarised fields due to some 'cross-talk' between the two excited linearly polarised resonances. Figure $2 \mathrm{~b}$ describes the wavelength-dependent polarisation state of the transmitted light. While this cross-talk reduces the conversion efficiency (Figure $2 \mathrm{~b}$ (black)), the structure remains capable of sufficient retardance to exhibit spin-to-orbit coupling of wavelengths $(\lambda)$ near $780 \mathrm{~nm}$, which has not been shown before in a plasmonic device or any structure with $\lambda / 30$ thickness.

The inhomogeneous plasmonic nano-antenna array is fabricated on a transparent substrate, consisting of a thin $(23 \mathrm{~nm})$ ITO layer on glass. The ITO layer does not affect the optical properties of the device, but serves as a charge dissipation layer during electron beam lithography. Electron beam lithography is performed using a $30 \mathrm{keV}$ Raith PIONEER system and a positive resist bilayer. Metal deposition and resist removal follow standard lift-off procedures, although it should be noted that no binding layer between the gold and ITO layers is necessary. The deposited gold layer was measured to be $27 \pm 3 \mathrm{~nm}$ thick. Scanning electron microscope images of the fabricated sample (Figure 2c) show a very uniform, rotationally symmetric metasurface. The nano-antenna array is locally uniform with an approximate lattice constant of $375 \mathrm{~nm}$. This results in the exceptionally small central singularity given by the diameter of the innermost ring $(750 \mathrm{~nm})$, significantly smaller than that with conventional liquid crystal technologies $(\sim 50 \mu \mathrm{m})$.

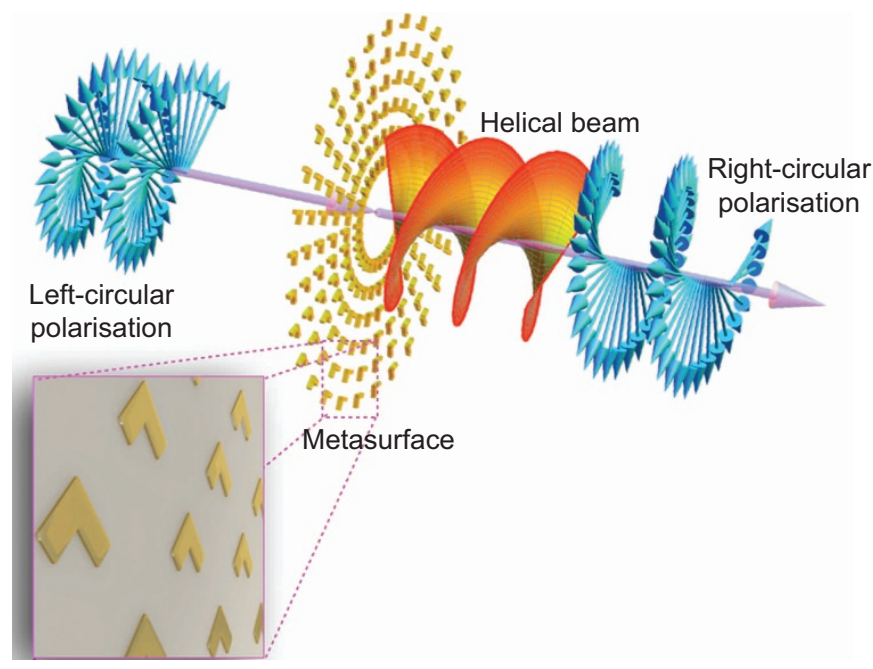

Figure 1 Schematic representation of spin-to-orbit coupling through a metasurface. The polarisation of the incident light is switched from left-handed circular to right-handed circular, giving the beam an OAM value of 2 due to conservation of angular momentum. Inset: locally, the circularly symmetric nano-antennas can be approximated as a uniform array, simplifying device modeling. OAM, orbital angular momentum. 

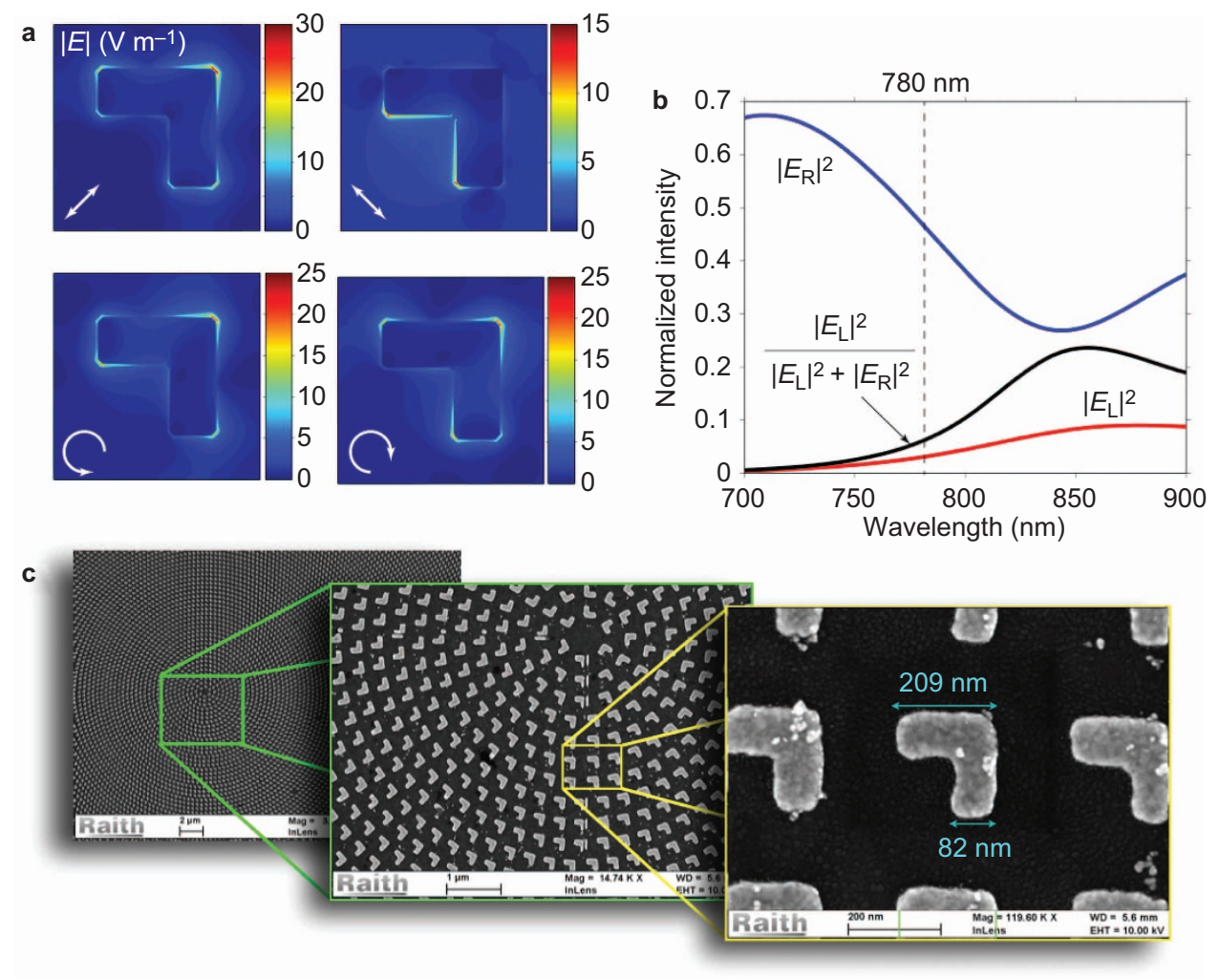

Figure 2 Structure and optical properties of the fabricated metasurface. (a) Simulated near-field distribution of the nano-antenna's resonances for different input polarisations. The anti-diagonal and diagonal linear polarisations excite two different modes, giving the metasurface its birefringent characteristics. The lower insets are the simulated near-field distributions for right and left-circularly polarised beams, respectively. (b) Calculated intensity of the transmitted right-circularly polarised (unconverted) beam, blue curve, and left-circularly polarised (converted) beam, red curve, for different wavelengths. The fields are normalized to the intensity of the incident beam. The conversion efficiency is shown as the solid black curve, which indicates an efficiency of $\sim 6 \%$ at a wavelength of $780 \mathrm{~nm}$. The dimensions of the fabricated sample are used for all simulations. (c) SEM images of the fabricated metasurface, consisting of an inhomogeneous array of L-shaped gold nano-antennas. The nano-antenna's arms are $210 \pm 5 \mathrm{~nm}$ long, $85 \pm 5 \mathrm{~nm}$ wide and $27 \pm 3 \mathrm{~nm}$ thick. Fewer than $1 \mathrm{in} 5000$ nano-antennas were lost in fabrication, resulting in a highly uniform metasurface. SEM, scanning electron microscope.

\section{RESULTS AND DISCUSSION}

We confirm OAM state generation by transmission measurements of circularly polarised light at $780 \mathrm{~nm}$. We isolate the converted part of the optical beam, which has opposite circular polarisation to any unconverted light, by a quarter-wave plate and a polariser. Figure 3a shows the intensity distribution of the converted optical beam. The preservation of the doughnut shape in both the near- and far-field confirms the existence of an optical vortex at the origin, where the singular point is stable throughout propagation. The multiple concentric rings are due to the excitation of higher order radial modes, which has been studied in detail elsewhere. ${ }^{22}$ Interference patterns between the converted light and either planar or spherical waves are imaged onto a charge coupled device (CCD) camera to determine the OAM value of the converted beam (Figure $3 \mathrm{~b}$ ) showing a double pitch-fork and double helix, respectively. The former confirms that the converted beam carries an OAM value of $2 \hbar$, based on the number of branches stemming from the singularity. Switching the incident polarisation from left-circular to right-circular does not change the output intensity pattern, but rather the OAM sign, changing the twisting direction of the helical wave-front and flipping the orientation of the fork fringes accordingly. We repeated the experiment for several wavelengths from $760 \mathrm{~nm}$ to $780 \mathrm{~nm}$, confirming broadband performance with efficiency increasing from $0.1 \%$ to $3 \%$ with increasing wavelength. This trend is consistent with, but approximately half of the conversion efficiency estimated by the simulations in Figure $2 b$.
Efficiencies as large as $20 \%$ are predicted by the simulations at slightly longer wavelengths $(\sim 850 \mathrm{~nm})$ and could be improved by refinements to the design and fabrication of the nano-antennas.

This demonstrates that a metasurface with $q=1$ induces OAM of \pm 2 on the converted beam, but the operating principle can be generalised to produce any integer value of OAM. The nano-antennas' arrangement provides the array with a well-defined integer or half-integer $q$. Based on the Pancharatnam-Berry phase, such a device introduces OAM of value $\pm 2 q$ to the outgoing beam. However, as such an array is not cylindrically symmetric, there will also be an exchange of angular momentum $|2 q-1| \hbar$ per photon between the array and the incident light.

\section{CONCLUSIONS}

In conclusion, we have demonstrated optical spin-to-orbit conversion at visible wavelengths in an ultrathin metasurface, consisting of a space-variant array of gold nano-antennas. This device is capable of generating light carrying OAM from light initially carrying only SAM, operating on the principle of the Pancharatnam-Berry phase. The metasurface is designed to possess a topological charge of one, thus introducing a space-variant optical phase onto the beam and giving it an OAM value of two, with the sign depending on the input polarisation state. This scheme provides a novel way to generate OAM in optical beams in the visible wavelength range, using ultrathin metamaterials suitable for integrated photonics. These ultrathin devices 
a

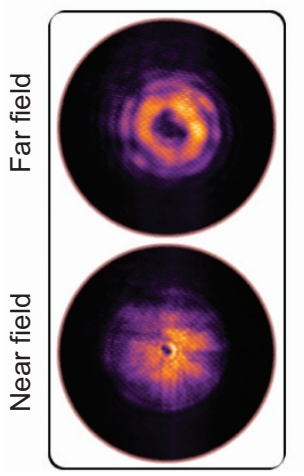

b

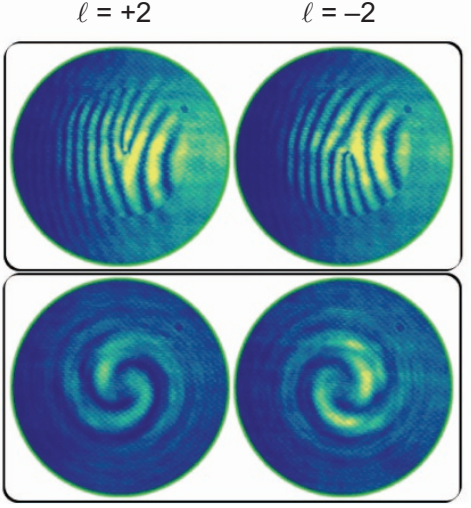

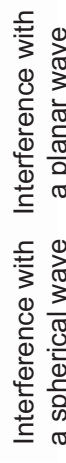

Figure 3 Intensity distribution and observed interference of the beam generated by the metasurface. (a) The intensity distribution of the converted component of the transmitted light. Upper and lower insets show the intensity at the far and near-field of the sample, respectively. The central null field at the origin results from the phase singularity. (b) The interference pattern of the generated beams with planar and spherical waves, respectively. The left and right insets correspond to left and right circularly polarised input beams. The number of branches of the fork fringes and lobes of the helices reveal that the transmitted beams possess $\mathrm{OAM}$ of $\ell=+2$ and $\ell=-2$, respectively. OAM, orbital angular momentum.

will allow a range of existing photonics technologies to be enhanced with OAM-based functionality, ${ }^{23,24}$ including spectroscopic and imaging applications for medical diagnostics, ${ }^{25}$ chemical analysis and astronomy; ${ }^{26}$ greater information encoding on individual photons in quantum communication systems, ${ }^{27}$ ultrasensitive devices $^{28}$ and potentially even inducing OAM on ultrashort pulses that otherwise would be disassociated by birefringent materials with even wavelength-order thickness. ${ }^{29}$

\section{AUTHOR CONTRIBUTIONS}

EK conceived the idea; IDL and HQ performed the structure's design and numerical analysis; SAS fabricated the sample; EK, JU and IDL performed the experiment; EK analyzed the data; RWB supervised all aspects of the project. All authors contributed to the text of the manuscript.

\section{ACKNOWLEDGMENTS}

The authors acknowledge the support of the Canada Excellence Research Chairs Program. We also acknowledge useful discussion with Anthony Olivieri concerning the bi-layer resist and lift-off processes. RWB was supported by the DARPA InPho program.

1 Allen L, Barnett SM, Padgett MJ. Optical Angular Momentum. Bristol: Institute of Physics; 2003.

2 Mair A, Vaziri A, Weihs G, Zeilinger A. Entanglement of the orbital angular momentum states of photons. Nature 2001; 412: 313-316.
3 Marrucci L, Manzo C, Paparo D. Optical spin-to-orbital angular momentum conversion in inhomogeneous anisotropic media. Phys Rev Lett 2006; 96: 163905.

4 Berry MV. The adiabatic phase and Pancharatnam's phase for polarized light. J Mod Opt 1987; 34: 1401-1407.

5 Bhandari R. Polarization of light and topological phases. Phys Rep 1997; 281: 1-64.

6 Bomzon Z, Biener G, Kleiner V, Hasman E. Space-variant Pancharatnam-Berry phase optical elements with computer-generated subwavelength gratings. Opt Lett 2002; 27: 1141-1143.

7 Marrucci L, Manzo C, Paparo D. Pancharatnam-Berry phase optical elements for wavefront shaping in the visible domain: switchable helical modes generation. Appl Phys Lett 2006; 88: 221102.

8 Park SR, Han J, Kim C, Koh YY, Kim C et al. Chiral orbital-angular momentum in the surface states of $\mathrm{Bi}_{2} \mathrm{Se}_{3}$. Phys Rev Lett 2012; 108: 046805.

9 D'Ambrosio V, Nagali E, Walborn SP, Aolita L, Slussarenko S et al. Complete experimental toolbox for alignment-free quantum communication. Nat Commun 2012; 3: 961.

10 Biener G, Niv A, Kleiner V, Hasman E. Formation of helical beams by use of Pancharatnam-Berry phase optical elements. Opt Lett 2002; 27: 1875-1877.

11 Cardano F, Karimi E, Slussarenko S, Marrucci L, de Lisio C et al. Polarization pattern of vector vortex beams generated by $q$-plates with different topological charges. Appl Opt 2012; 51: C1-C6.

12 Zheludev NI, Kivshar YS. From metamaterials to metadevices. Nat Mater 2012; 11 : 917-924.

13 Gansel JK, Thiel M, Rill MS, Decker M, Bade K et al. Gold helix photonic metamaterial as broadband circular polarizer. Science 2009; 325: 1513-1515.

14 Aieta F, Genevet P, Kats MA, Yu NF, Blanchard R et al. Aberration-free ultrathin flat lenses and axicons at telecom wavelengths based on plasmonic metasurfaces. Nano Lett 2012; 12: 4932-4936.

15 Genevet P, Yu NF, Aieta F, Lin J, Kats MA et al. Ultra-thin plasmonic optical vortex plate based on phase discontinuities. Appl Phys Lett 2012; 100: 013101.

16 Yu NF, Genevet P, Kats MA, Aieta F, Tetienne JP et al. Light Propagation with phase discontinuities: generalized laws of reflection and refraction. Science 2011; 334 333-337.

17 Lawrence N, Trevino J, dal Negro L. Control of optical orbital angular momentum by Vogel spiral arrays of metallic nanoparticles. Opt Lett 2012; 37: 5076-5078.

18 Williams MD, Coles MM, Saadi K, Bradshaw DS, Andrews DL. Optical vortex generation from molecular chromophore arrays. Phys Rev Lett 2013; 111: 153603.

19 Beth RA. Mechanical detection and measurement of the angular momentum of light. Phys Rev 1936; 50: 115-125.

20 Karimi E, Zito G, Piccirillo B, Marrucci L, Santamato E. Hypergeometric-Gaussian modes. Opt Lett 2007; 32: 3053-3055.

21 Palik ED. Handbook of Optical Constants of Solids. New York: Academic Press; 1985.

22 Karimi E, Piccirillo B, Marrucci L, Santamato E. Light propagation in a birefringent plate with topological charge. Opt Lett 2009; 34: 1225-1227.

23 Litchinitser NM. Structured light meets structured matter. Science 2012; 337 1054-1055.

24 Kildishev AV, Boltasseva A, Shalaev VM. Planar photonics with metasurfaces. Science 2013; 339: 1232009.

25 Hell SW. Far-field optical nanoscopy. Science 2007; 316: 1153-1158.

26 Foo G, Palacios DM, Swartzlander GA. Optical vortex coronagraph. Opt Lett2005; 30 : 3308-3310.

27 Molina-Terriza G, Torres JP, Torner L. Twisted photons. Nat Phys 2007; 3: 305 310.

28 D’Ambrosio V, Spagnolo N, del Re L, Slussarenko S, Li Y et al. Photonic polarization gears for ultra-sensitive angular measurements. Nat Commun 2013; 4: 2432.

29 Karimi E, Altucci C, Tosa V, Velotta R, Marrucci L. Influence of generalized focusing of few-cycle Gaussian pulses in attosecond pulse generation. Opt Exp 2013; 21 : 24991-24999.

(i)

This work is licensed under a Creative Commons Attribution 3.0 Unported License. The images or other third party material in this article are included in the article's Creative Commons license, unless indicated otherwise in the credit line; if the material is not included under the Creative Commons license, users will need to obtain permission from the license holder to reproduce the material. To view a copy of this license, visit http://creativecommons.org/ licenses/by/3.0/ 